\title{
Metodología Para La Caracterización De Paisajes Fluviales: Aplicación A La Cuenca Hidrográfica Del Tajo (España Central)
}

\section{Methodology For Characterization Of Fluvial Landscapes: Aplicattion To The Tagus Basin (Central Spain)}

\author{
Pedro Molina Holgado \\ Universidad Autonoma de Madrid
}

Ana Belén Berrocal Menárguez

Universidad Politécnica de Madrid

\begin{abstract}
Resumen: Los espacios fluviales son áreas de elevado interés natural y cultural. Desde hace décadas son objeto prioritario de investigaciones de diversa naturaleza y, más recientemente, de programas de gestión específicos que asumen su importancia para el mantenimiento de la diversidad ecológica y paisajística. Como contribución al conocimiento de estos ámbitos de especial valor, en este trabajo se presenta una metodología para la caracterización de paisajes fluviales. Se basa en la identificación de diferentes tipos de paisajes considerando 45 ítems agrupados en 7 grandes categorías. La unidad básica sobre la que se realiza la caracterización son tramos o sectores fluviales, delimitados principalmente en función de criterios morfológicos, hidrológicos o, en algunos casos, relacionados con factores antrópicos.
\end{abstract}

Palabras clave: Paisajes fluviales. Tramos fluviales. Caracterización del paisaje fluvial. Cuenca hidrográfica del Tajo

Resumo: Os espaços fluviais são áreas de elevado interesse natural e cultural. Há décadas têm sido objeto prioritário em investigações diversas e, mais recentemente, de programas de gestão específicos importantes para a manutenção da diversidade ecológica e paisagística. Como contribuição a compreensão dessas áreas de especial valor, este trabalho apresenta uma metodologia para a caracterização de paisagens fluviais. Baseia-se na identificação dos diferentes tipos de paisagens, considerados a partir de 45 itens agrupados em sete grandes categorias. A unidade básica sobre a qual se realiza a caracterização são trechos de rio ou setores fluviais, definidos principalmente em função de critérios morfológicos, hidrológicos ou, em alguns casos, relacionados a fatores antrópicos.

Palavras-chave: Paisagens fluviais. Setores fluviais. Caracterização da paisagem fluvial. Bacia hidrográfica do rio Tejo.

Abstract: The riverine spaces comprehend areas of great natural and cultural interest. They have been prioritized for many decades by several researches and, more recently, by specific management programs that are important to the maintenance of the ecological and landscape diversity. As a contribution to the understanding of these high value areas, this paper presents a methodology for the characterization of riverine landscapes. It is based on the identification of different types of landscapes, considering 45 items grouped into seven broad categories. The basic unit on which this characterization is 
based are river sections or sectors, defined primarily in terms of morphological and hydrological criteria, or in some cases as related to anthropogenic factors.

Keywords: Riverine landscape. Fluvial sectors. Fluvial landscape characterization. Tagus basin.

\section{INTRODUCCION}

El paisaje sintetiza la relación que las comunidades humanas han mantenido con su entorno en el espacio y en el tiempo (MARTÍNEZ DE PISÓN, 2009; ANTROP, 2005; CARAPINHA, 2009). En su entendimiento y análisis participan ciencia, como realidad concreta y cuantificable, y percepción, como expresión de visiones subjetivas y sentimientos (ORTEGA, 2008). Posee, por tanto, varias referencias: su propia morfología tangible, la percepción de quien lo vive u observa, y su análisis o representación desde la ciencia y la cultura (MARTÍNEZ DE PISÓN, 2010). El paisaje es también por este motivo una experiencia sensorial, estética o artística (MATA, 2006; SAULE-SORBÉ, 2006; RENES, 2009) y por ello está estrechamente vinculado a los procesos que han creado la memoria histórica y la identidad nacional de los pueblos (ORTEGA, 2005).

Los paisajes vinculados a los cursos fluviales poseen características propias y valores específicos, diferenciales respecto a otros, debido a que el agua es el elemento que organiza su conectividad espacial y estructura la vida y las propias formas de ocupación del territorio (HORDEN; PURCELL, 2001), como bien puede observarse en numerosas civilizaciones antiguas y culturas tradicionales (GRANERO, 2008; LAUREANO, 2005). Por este motivo, los paisajes fluviales han sido y aún son hoy objeto de intensas presiones antrópicas que, con frecuencia, han provocado una intensa modificación y alteración de sus estructuras naturales (CASIMIRO; SANCHEZ; COSTILLO,
2003; NAIMAN et al.,1993; KNOPF et al., 1988). Como señalan Vadillo y Molina (2010), los paisajes fluviales de Europa han experimentado una fuerte degradación (YON; TENDRON, 1981) y, en España, se estima que la desaparición de cauces naturales en el periodo 1987-2000 alcanzó un valor del 12\% (7.508 ha) (GONZÁLEZ; DE LA LASTRA, 2007).

El entendimiento global del paisaje requiere la comprensión de sus partes y, por ello, el análisis de sus componentes y estructura, el reconocimiento de su singularidad, riqueza y valor en el sentido indicado en el informe del Observatorio de la Sostenibilidad en España relativo al patrimonio natural, cultural y paisajístico (JIMÉNEZ, 2009). En esta línea, el Convenio Europeo del Paisaje, firmado en Florencia en 2000, pone de manifiesto la importancia de la gestión y protección de los paisajes y exige la creación de instrumentos que permitan caracterizarlos y valorarlos, como ya se ha realizado en España y en el conjunto de Europa a media escala (MATA SANZ et al. 2003; COUNCIL OF EUROPE, 2006). En este contexto se sitúa este trabajo: desarrolla un método para la caracterización de la dimensión paisajística del territorio fluvial, como configuración formal y realidad tangible.

Esta metodología de caracterización, cuyo ámbito de análisis se sitúa en los paisajes fluviales de la ribera del río Tajo en las provincias de Guadalajara, Madrid y Toledo (España Central), se plantea por su interés intrínseco, como elemento para el mejor conocimiento del paisaje, pero también por su interés instrumental de cara a su posible aplicación en la gestión global del territorio. 


\section{METODOLOGÍA PARA LA CARACTERIZACIÓN DE PAISAJES FLUVIALES}

\subsection{Consideraciones generales}

El análisis de los paisajes fluviales que aquí se presenta, vinculado a un trabajo de investigación más amplio dedicado a la cuenca hidrográfica del río Tajo (MOLINA; SANZ; MATA, 2010), se realiza de manera cualitativa y semicuantitativa sobre sectores o tramos fluviales (fluvial sectors) de dimensiones concretas de los ríos Tajo y Jarama. En la medida en que el paisaje en general y el paisaje fluvial en particular se conforman como una síntesis de naturaleza y cultura (BETHEMONT, 1999), considera criterios físicos y humanos: son 45 criterios específicos de este tipo de medios en general, y de los cursos de la Cuenca del Tajo en particular.

Este análisis analiza los elementos del paisaje y este en su conjunto en cada uno de los tramos que se identifican en la zona seleccionada para la aplicación del método. La metodología de análisis se basa en algunos ensayos llevados a cabo con anterioridad por los autores (MOLINA; BERROCAL, 2006a; MOLINA; BERROCAL, 2006b; MOLINA, 2007). Por su estructura y objetivos, puede vincularse a las propuestas metodológicas de diversos trabajos que construyen taxonomías cuyo objetivo es la caracterización y valoración de espacios fluviales de España, en muchos casos de acuerdo con las exigencias establecidas en la Directiva Marco del Agua (GONZÁLEZ DEL TÁNAGO; GARCÍA DE JALÓN; LARA; GARILLETI, 2006; GONZÁLEZ DEL TÁNAGO; GARCÍA DE JALÓN, 2006, 2007; OLLERO et al., 2004); también con otros ensayos de clasificación referidos a la calidad ambiental de las riberas fluviales (MUNNÉ; SOLÀ; PRAT, 1998; SUÁREZ; VIDAL-ABARCA, 2000;
SUÁREZ et al., 2002) o a su organización geomorfológica (DÍAZ; OLLERO, 2005).

La tipificación de los paisajes fluviales que se deriva de la aplicación de esta metodología es aplicable a todos los cursos de la Península Ibérica. Puede ser igualmente aplicable a otros espacios fluviales situados en diferentes contextos naturales y culturales, adaptando los criterios de caracterización a esas realidades paisajísticas. La Figura 1 muestra la estructura básica del método de caracterización desarrollado.

Figura 1 - Fases del desarrollo de la metodología de caracterización de paisajes fluviales

A. IDENTIFICACIÓN DE TRAMOS

Fotointerpretacion, corrección y mejora de límites en campo

\begin{tabular}{|c|}
\hline B. CARACTERIZACIÓN \\
\hline B1. Establecimiento de criterios y clases de valor \\
\hline B2. Distribución de pesos/criterio \\
\hline $\begin{array}{c}\text { Consulta de fuentes, documentales y } \\
\text { cartográficas, toma de dados en campo }\end{array}$ \\
\hline \multicolumn{2}{|c|}{ B4. Creación de bases de dados } \\
\hline B5. Análisis de similitud de tramos \\
B6. Análisis UPGMA \\
RESULTADOS \\
FLUVIALES \\
\hline IDENTIFICACIÓN DES DAJES
\end{tabular}

Org.: Autores 


\section{2 Ámbito de aplicación de la metodología de caracterización}

El área seleccionada para desarrollar y aplicar esta metodología de caracterización e identificación de paisajes fluviales abarca un extenso sector de la ribera del río Tajo, entre las localidades de Peralejos de las Truchas (Guadalajara) y el embalse de Valdecañas (Cáceres). Incluye asimismo el tramo bajo del río Jarama, desde la desembocadura de su afluente el río Manzanares hasta su desagüe en el río Tajo (Figura 2). Esta zona se sitúa entre las cotas 312-1.056 $\mathrm{m}$ y drena una superficie de cuenca de $36.550 \mathrm{~km}^{2}$, valor que representa el 65,68\% de la extensión total de la cuenca del Tajo en España $\left(55.645 \mathrm{~km}^{2}\right)$. El sector analizado abarca un total de $640,76 \mathrm{~km}$ de cauce, más del $60 \%$ de la longitud de este curso en España, y $525 \mathrm{~km}$ de valle.

Figura 2 - Zona de aplicación de la metodología en la Cuenca del TajoEn color marrón claro, el sector español de la cuenca (55.870 km²), en marrón oscuro el sector portugués $\left(27.808 \mathrm{~km}^{2}\right)$. Las flechas rojas muestran la los límites de la zona analizada en la ribera del Tajo. Las flechas verdes los límites en la ribera del río Jarama.

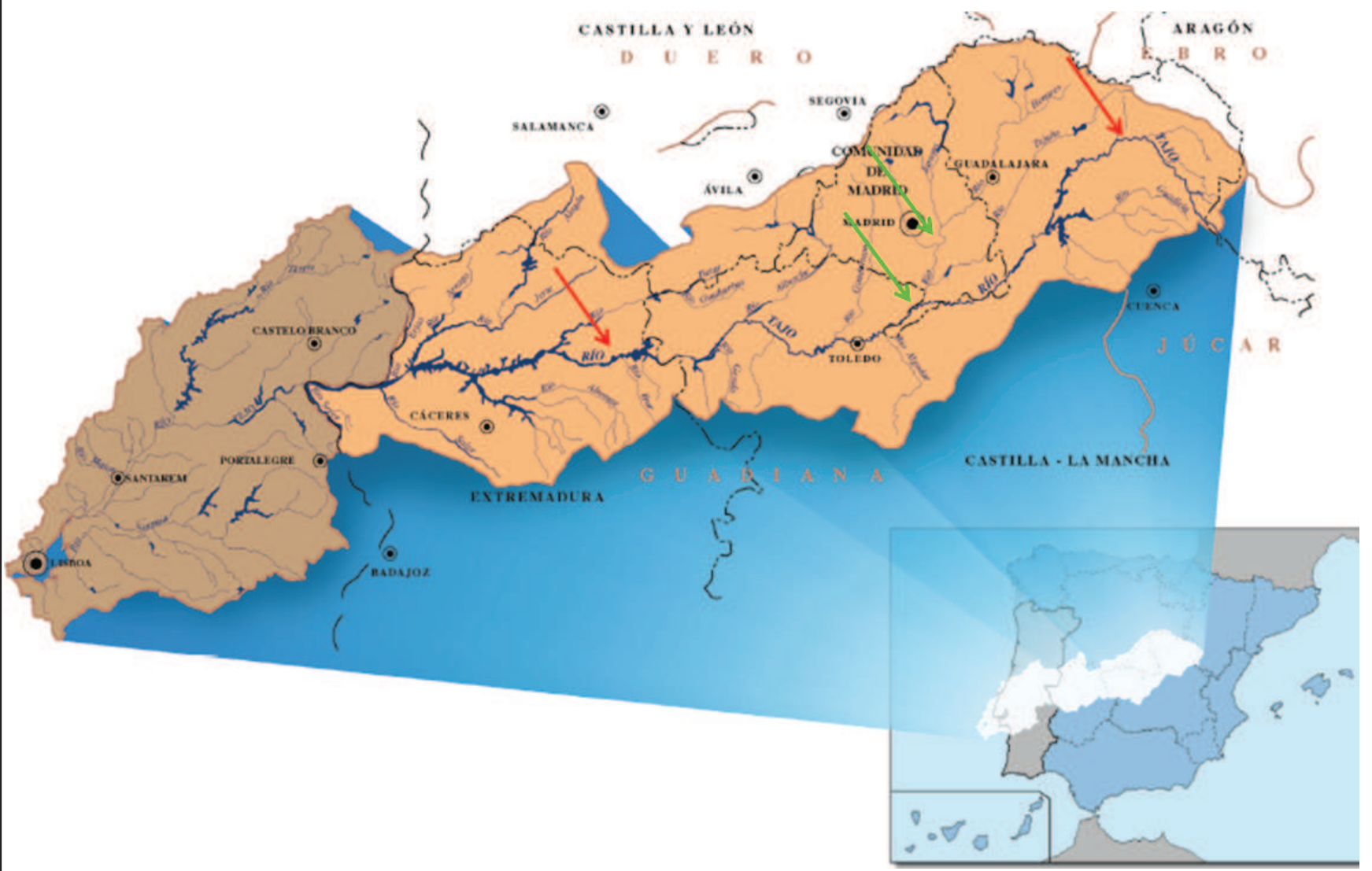

Fuente: CONFEDERACIÓN HIDROGRÁFICA DEL TAJO, 2002.

La elección de los ríos Tajo y Jarama para poner a punto esta metodología se debe a de su representatividad a la escala de cuenca hidrográfica. Son muchos más los cursos fluviales existentes pero los dos cauces elegidos se integran en una amplia variedad de paisajes zonales. No se incluyen en este análisis los tramos genuinamente montañosos situados a más de $1.000 \mathrm{~m}$ : a esta escala de análisis, por su reducida amplitud, los paisajes fluviales de montaña no pueden discriminarse de aquellos otros en los que se sitúan. 


\subsection{Identificación de tramos}

Los criterios básicos para la identificación de tramos fluviales, las unidades funcionales de análisis sobre las que se realiza la caracterización, se relacionan con sus características geomorfológicas e hidrológicas: la morfología del fondo y en algunos casos vertientes del valle, las pendientes del cauce y del valle, sinuosidad y dinámica activa del canal de bankfull, la aportación media anual y el tipo de régimen rápido o lento, entre otros, son factores ecopaisajísticos que se manifiestan con claridad en el territorio y permiten realizar una primera identificación.

Los tramos incluyen, por tanto, unos límites longitudinales que permiten compartimentar el continuo fluvial, establecidos en función de los criterios ya señalados, y también unos límites laterales que permiten separar los espacios fluviales de los zonales o estrictamente terrestres. Estos últimos se establecen en función de la importancia del agua como elemento estructurante del paisaje y del territorio (BETHEMON;PIEGAY,1998; BETHEMON et al., 2006; MALANSON, 1993).

En efecto, una cuestión importante para el análisis de los paisajes fluviales es su separación de los paisajes zonales colindantes, de carácter no fluvial (campiñas, páramos, penillanuras, etc.). Como ya se ha indicado, se considera que forman parte del dominio de los paisajes fluviales los sectores de los fondos de valle cuyas características básicas están determinadas por la abundancia de agua, ya sea ésta de origen natural o antrópico. Son los sectores de las llanuras aluviales que se benefician de la aportación del subálveo, del desbordamiento natural de los cauces o del riego. Son tierras fértiles, asiento de especies y comunidades azonales edafohigrófilas (bosques aluviales o sotos) que colonizaron los depósitos aluviales más bajos, las llanuras de inundación y, en su caso, la terraza inmediatamente superior, sustituidas actualmente en gran parte por cultivos de regadío. Los límites de estos paisajes se localizan allí donde el agua pierde importancia como elemento organizador. Es un límite a veces nítido, pero en ocasiones difícil de establecer. Las fronteras entre estos paisajes de agua y los característicos de las vertientes son en muchos casos graduales.

Los criterios de delimitación, las dominantes paisajísticas de percepción directa, pueden variar en algunos casos. En la zona analizada, los contrastes geomorfológicos que se producen en breves espacios son notables; sin embargo, en términos paisajísticos, la identificación de tramos no se basa únicamente en estos criterios morfoestructurales y litológicos. En particular, esto se aprecia en los entornos urbanos, donde la interfase ríociudad constituye siempre un tipo de paisaje específico (PELLICER, 2002), aunque lo natural siempre subyace.

La identificación de tramos busca, fundamentalmente, coherencia territorial y paisajística, no uniformidad en la longitud de los sectores reconocidos. Por este motivo, las longitudes de los tramos identificados pueden presentar diferencias notables, acusadas en tramos montanos y poco notables en los paisajes fluviales de las grandes cuencas debido a la mayor uniformidad litológica y morfoestructural del sector central de la cuenca.

Este análisis se ha realizado mediante interpretación de imágenes aéreas a diversas escalas, consulta de cartografía topográfica (Mapa Topográfico del Instituto Geográfico Nacional, E. 1:25.000) y consulta de cartografía temática (Mapa Geológico de España, ITGME, E. 1:50.000; Mapa Forestal de España, DGCN, E.1:200.000, etc). Los resultados de los trabajos de gabinete, contrastados en campo, han permitido diferenciar en esta primera fase 38 tramos fluviales sobre los que se ha realizado el análisis paramétrico (Tabela 1). 


\begin{tabular}{|c|c|c|c|c|c|c|c|}
\hline 焉岂 & 8 & 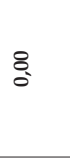 & $\begin{array}{l}9 \\
\text { g. } \\
\delta \\
\text { in }\end{array}$ & 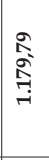 & 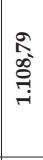 & 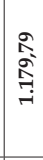 & 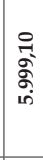 \\
\hline 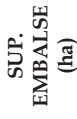 & & & 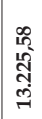 & & & ' & 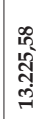 \\
\hline 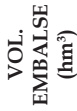 & & & 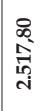 & & & & \\
\hline 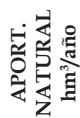 & $\begin{array}{l}\tilde{Z} \\
\tilde{J}\end{array}$ & & $\begin{array}{l}0 \\
0 \\
0 \\
0 \\
\sigma \\
7\end{array}$ & 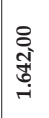 & 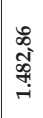 & 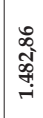 & 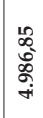 \\
\hline 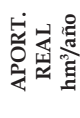 & 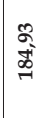 & & 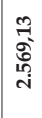 & 荧 & $\begin{array}{l}8 \\
0 \\
0 \\
\infty \\
\infty\end{array}$ & : & $\begin{array}{l}9 \\
0 \\
8 \\
0.0\end{array}$ \\
\hline 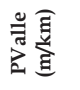 & ন্ & ปั) & & Бू & 点 & & \\
\hline 总言 & \begin{tabular}{|l}
$\mathbb{I}$ \\
$-\pi$
\end{tabular} & वे & & 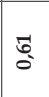 & F & & \\
\hline - - 咅 & & & & $\stackrel{\sqrt[5]{2}}{-}$ & $\stackrel{0}{a}$ & & \\
\hline 怔 & ๙ิ & स्त & $\begin{array}{l}\text { 㟢 } \\
\text { 亲 }\end{array}$ & $\begin{array}{l}\text { İ } \\
\text { J̇ }\end{array}$ & 焉 & 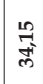 & 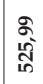 \\
\hline U西 & $\begin{array}{l}\text { స̃ } \\
\text { בे }\end{array}$ & 㐬 & $\begin{array}{l}\overrightarrow{5} \\
\overrightarrow{0}\end{array}$ & 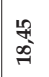 & 总 & $\mid \begin{array}{l}\infty \\
\infty \\
\infty \\
\infty \\
\infty\end{array}$ & 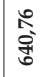 \\
\hline 起主 & 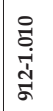 & 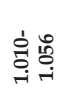 & 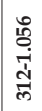 & 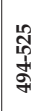 & 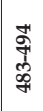 & 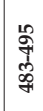 & 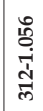 \\
\hline 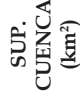 & & & 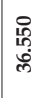 & 蛋 & 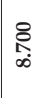 & $\begin{array}{l}\text { तु } \\
\text { तु }\end{array}$ & 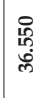 \\
\hline 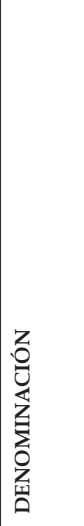 & 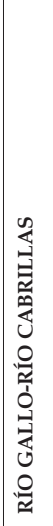 & 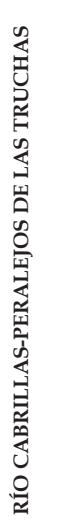 & 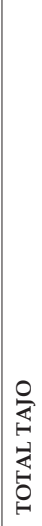 & 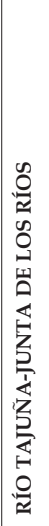 & 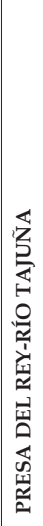 & 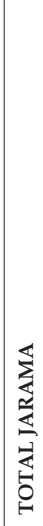 & 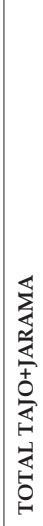 \\
\hline & 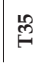 & 吊 & & $=$ & $\simeq$ & & \\
\hline
\end{tabular}

\subsection{Criterios y método de caracterización de tramos e identificación de tipos a partir del análisis paramétrico}

Los criterios considerados para el desarrollo de la caracterización paisajística y posterior identificación de tipos de paisaje tienen un carácter abierto que permite su aplicación a otros ámbitos fluviales no incluidos en el territorio objeto de este análisis. Son ítems que por su naturaleza pueden ser aplicados a fondos de valle y riberas situadas en otras grandes cuencas ibéricas o a cursos de otros ámbitos geográficos.

Las fases de trabajo seguidas en la caracterización han sido las siguientes:

- Fase 1. Establecimiento de criterios y clases de valor (Cuadros 1 y 2, Tabela 2)

-Fase 2. Distribución de pesos-criterio (Figuras 3 y 4 )

-Fase 3. Análisis de similitud de tramos (Figura 5)

- Fase 4. Representación gráfica de la similitud: identificación de tipos de paisajes (Figura 6).

Una vez delimitados los tramos fluviales, la primea fase de este método (Fase 1) ha consistido en la selección de los criterios de caracterización. En total se han considerado 45 ítems agrupados en 7 grandes categorías (Cuadro 1). 
Cuadro 1 - Distribución de los criterios de caracterización por categorías

\section{GRUPO I: RASGOS GENERALES (COROLÓGICOS)}

\section{GRUPO II: FACTORES HIDROLÓGICOS E HIDRÁULICOS}

\section{GRUPO III: FACTORES GEOMORFOLÓGICOS Y TOPOGRÁFICOS}

\section{GRUPO IV: VEGETACIÓN}

GRUPO V: AGROSISTEMA

\section{GRUPO VI: TRAMAS E INFRAESTRUCTURAS RURALES}

\section{GRUPO VII: POBLAMIENTO}

\begin{tabular}{|c|}
\hline PROVINCIA BIOGEOGRÁFICA \\
\hline SUBPROVINCIA BIOGEOGRÁFICA \\
\hline SECTOR BIOGEOGRÁFICO \\
\hline APORTACIÓN MEDIA ANUAL EN HM ${ }^{3}$ \\
\hline TAMAÑO DE LA CUENCA \\
\hline ESTACIONALIDAD DE LOS CAUDALES \\
\hline CALIDAD DEL AGUA \\
\hline GRADO DE REGULACIÓN \\
\hline APORTACIÓN REAL/APORTACIÓN NATURAL \\
\hline ALTERACIÓN EN LA DISTRIBUCIÓN DE CAUDALES \\
\hline GRADO DE INUNDACIÓN (VOLUMEN DE EMBALSE) \\
\hline EMBALSES-AZUDES (SUPERFICIE) \\
\hline PENDIENTE MEDIA DEL CAUCE \\
\hline PENDIENTE MEDIA DEL VALLE \\
\hline TIPO DE CANAL \\
\hline MOVILIDAD DEL CANAL \\
\hline NIVEL ALTITUDINAL \\
\hline MORFOLOGÍA DEL FONDO DE VALLE \\
\hline EXTENSIÓN LINEAL DEL FONDO DE VALLE (LLI+T1) \\
\hline MARCO GEOMORFOLÓGICO \\
\hline LITOLOGÍA DE LA CAJA \\
\hline MATERIALES DOMINANTES EN LA LLANURA DE INUNDACIÓN \\
\hline TIPO DE VEGETACIÓN \\
\hline COMUNIDADES VEGETALES \\
\hline ESTRUCTURA DE LA VEGETACIÓN \\
\hline EXTENSIÓN DE LA VEGETACIÓN \\
\hline CONTINUIDAD LINEAL DE LA VEGETACIÓN EN \% \\
\hline COMPLEJIDAD ESTRUCTURAL \\
\hline CONEXIÓN RIBERA-VEGA-VERTIENTE \\
\hline RIEGO \\
\hline TIPO DE REGADÍO \\
\hline TIPO DE CULTIVO \\
\hline GRADO DE INTENSIDAD PRODUCTIVA \\
\hline TAMAÑO DE LA EXPLOTACIÓN Y PARCELARIO \\
\hline CANALES \\
\hline VIARIO \\
\hline TRANSPORTE DE ENERGÍA \\
\hline TRAMOS URBANOS \\
\hline NÚCLEOS TRADICIONALES \\
\hline NÚCLEOS TRADICIONALES TIPO \\
\hline NÚCLEOS TRADICIONALES LOCALIZACIÓN \\
\hline NÚCLEOS DE COLONIZACIÓN \\
\hline NÚCLEOS DE COLONIZACIÓN TIPO \\
\hline POBLAMIENTO DISEMINADO \\
\hline POBLAMIENTO DISEMINADO TIPO \\
\hline $\begin{array}{l}\text { GRANDES SUPERFICIE PRODUCTIVAS, EQUIPAMIENTOS Y OTRAS } \\
\text { DOTACIONES }\end{array}$ \\
\hline
\end{tabular}

Org.: Autores 
Posteriormente se ha otorgado un peso a cada uno de estos ítems (Fase 2), de acuerdo con las opiniones formuladas por un panel de 6 expertos (geografía física, geografía agraria, ingeniería civilarquitectura, ecología-biología), siendo su cómputo global 296 puntos.

Además delos criterios decaracterización, la matriz incluye lo que se han denominado clases de valor. Estas clases tratan de evitar una exagerada desagregación de los valores de caracterización, intentando de esta manera salvar distancias irreales entre tipos de paisajes.

Cuadro 2 - Ejemplo de Criterios y clases. Item Aportación media anual en $\mathrm{hm}^{3}$

\begin{tabular}{|c|c|c|c|}
\hline \multirow{11}{*}{$\begin{array}{l}\text { APORTACIÓN } \\
\text { MEDIA ANUAL } \\
\text { EN hm }\end{array}$} & $<50$ & \multirow{11}{*}{$\begin{array}{c}\text { APORTACIÓN } \\
\text { MEDIA ANUAL } \\
\text { EN hm (CLASES) }\end{array}$} & $<250$ \\
\hline & $50-100$ & & \multirow{2}{*}{$250-500$} \\
\hline & $100-250$ & & \\
\hline & $250-500$ & & \multirow{2}{*}{$500-1000$} \\
\hline & $500-1.000$ & & \\
\hline & $1.000-2.000$ & & \multirow{2}{*}{$1.000-3.000$} \\
\hline & $2.000-3.000$ & & \\
\hline & $3.000-5.000$ & & \multirow{2}{*}{$3.000-7.000$} \\
\hline & $5.000-7.000$ & & \\
\hline & $7.000-10.000$ & & \multirow{2}{*}{$>7.000$} \\
\hline & $>10.000$ & & \\
\hline
\end{tabular}

Org.: Autores

Así, por ejemplo, el ítem Aportación media anual en $\mathrm{hm}^{3}$ (peso 10) identifica 11 niveles, mientras que su respectiva clase de valor los reúne en 6 , otorgando también, 10 puntos (Cuadro 2). Si no se hubiesen incluido estos factores de corrección se habría otorgado la misma distancia a la que existe entre dos tramos con aportaciones medias anuales próximas $\left(<500 \mathrm{hm}^{3} \mathrm{y}\right.$ $\left.500-1.000 \mathrm{hm}^{3}\right)$ y lejanas $\left(500-1000 \mathrm{hm}^{3-}>\right.$ 4.000). De haber valorado así este criterio se habría producido una dispersión que no se corresponde con la realidad de estos y otros paisajes fluviales. Estos factores de corrección o clases se han incluido en todos aquellos casos en los que se ha estimado que existía posibilidad de dispersión.

Si se observa la distribución gráfica de valores (296 puntos global) recogida globalmente en las Figuras 3 y 4 y de manera más detallada en la Tabela 2, los pesos muestran una distancia moderada entre sí $(x=45,2 \pm 17,8 ; n=5)$ si se excluyen de este análisis los pertenecientes a los grupos I y VII. Estas distancias aumentan considerablemente al incorporar los ítems pertenecientes al grupo VII (Poblamiento) $(x=42,3 \pm 20,7 ; n=7)$ debido al elevado peso global otorgado a este grupo de criterios. De manera intencionada se ha otorgado un valor global de 60 puntos (20,3\%) a este grupo (Poblamiento), de los cuales 25 puntos corresponden al ítem que discrimina los tramos fluviales urbanos de los no urbanos y suburbanos. Se ha considerado que era adecuado discriminar de manera clara este tipo de tramos ya que, en estos casos, sin duda, es lo urbano lo que articula el paisaje, imponiéndose como criterio sobre otros elementos del medio. Una explicación detallada relativa a los criterios de caracterización puede encontrarse en Molina, Sanz y Mata (2010).

Una vez realizada la identificación de criterios (Fase 1) y la distribución de pesos (Fase 2) se ha procedido a la identificación de los tipos de paisajes fluviales (Fases 3 y 4). Para ellos se ha obtenido un índice o medida de similitud para todos los tramos analizados, en este caso el cuadrado de la distancia euclidiana; posteriormente, mediante la aplicación de un análisis aglomerativo cluster UPGMA vecino más próximo), se han identificado las clases o tipos de paisajes fluviales en función de la mayor o menor afinidad observada. 
Figura 3 - Distribución porcentual de la importancia de los factores por grupos de caracterización de paisajes fluviales en la Cuenca Hidrográfica del Tajo

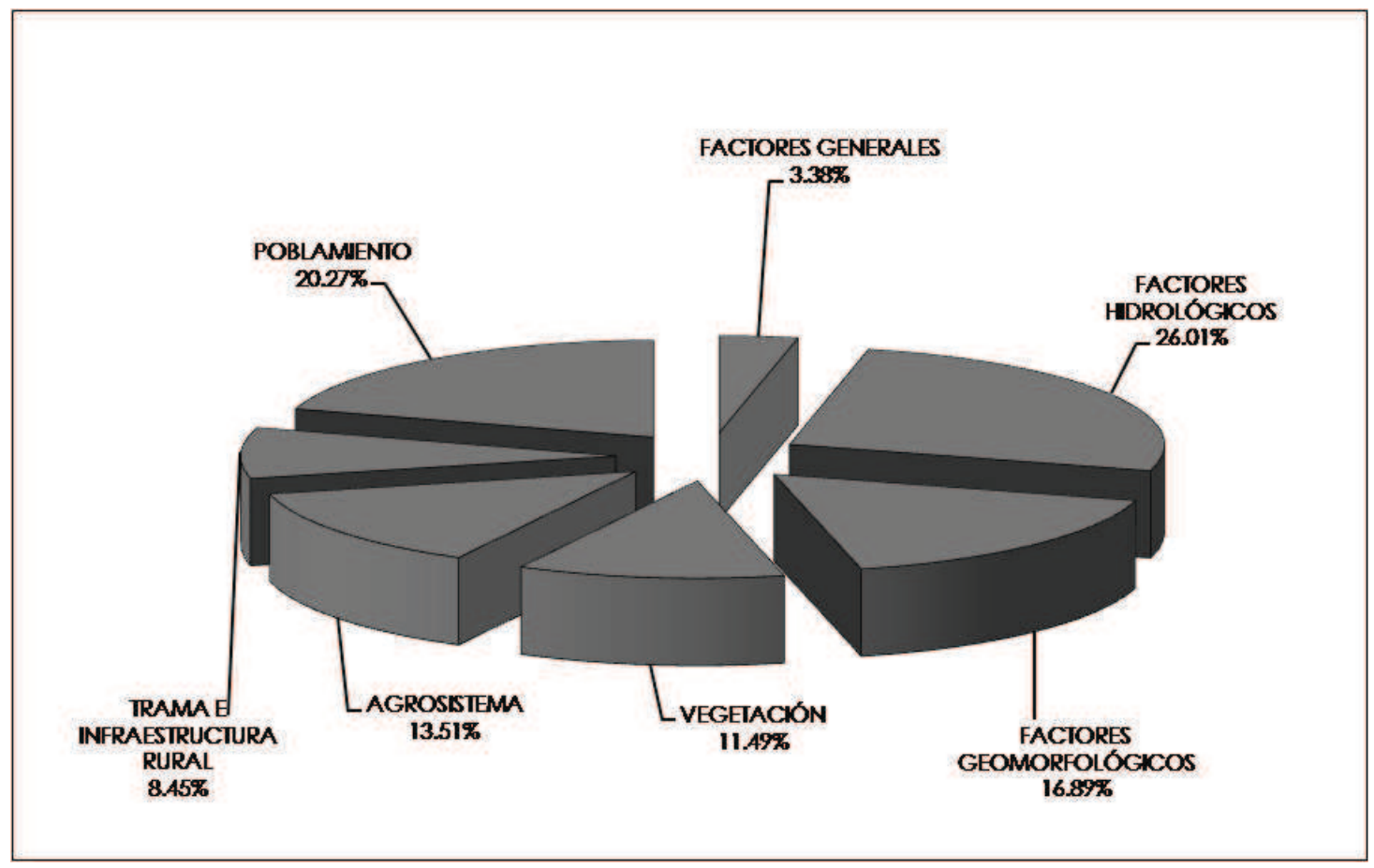

Org.: Autores

Figura 4 - Peso por grupos de los factores de caracterización de paisajes fluviales en la Cuenca Hidrográfica del Tajo

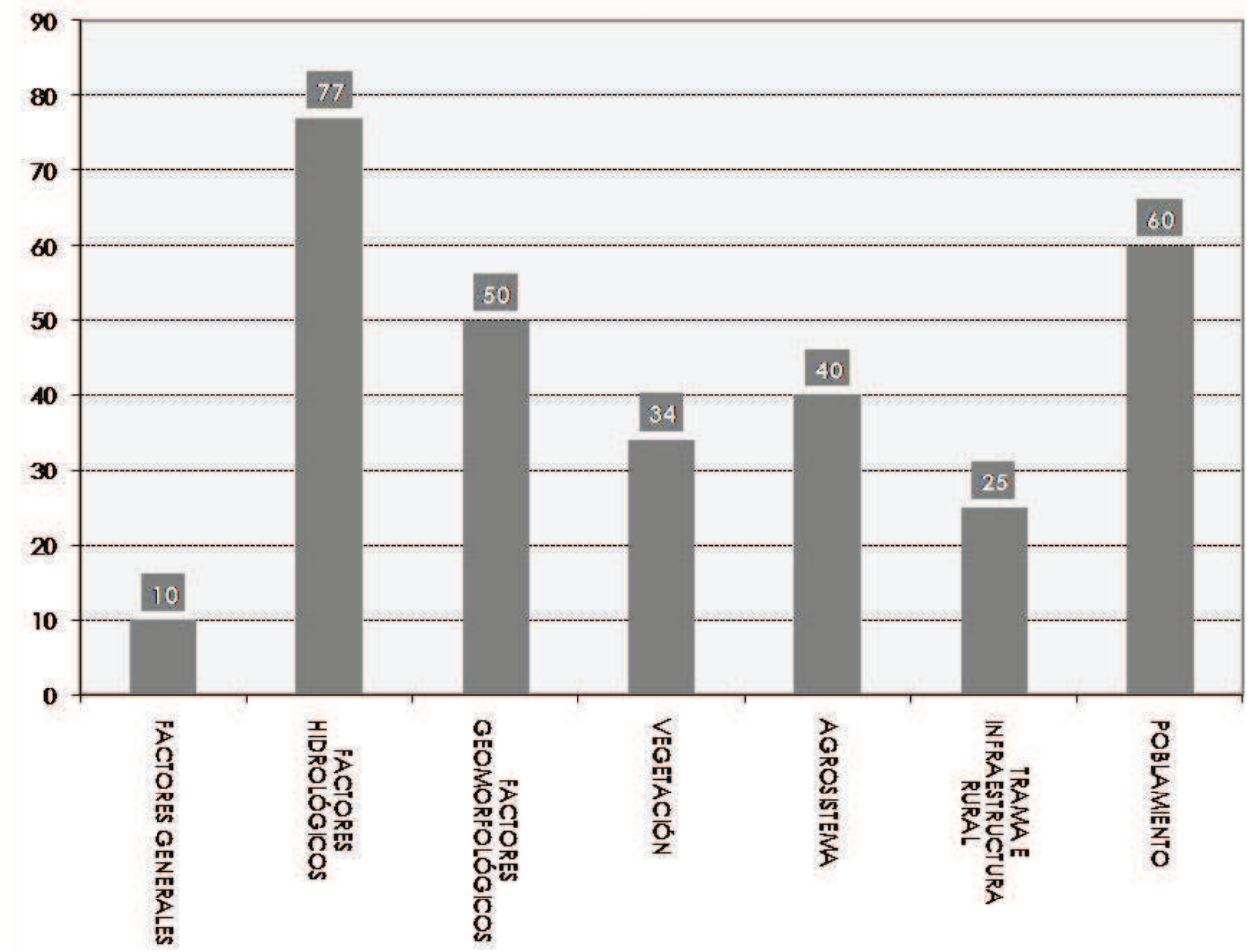

Org.: Autores 
Tabela 2 - Factores ecopaisajísticos para la caracterización de paisajes fluviales en la Cuenca Hidrográfica del Tajo

\begin{tabular}{|c|c|c|}
\hline PESO & FACTORES & PARÁMETROS \\
\hline 10 & \multicolumn{2}{|c|}{ RASGOS GENERALES } \\
\hline \multirow{2}{*}{2} & \multirow{2}{*}{ REGIÓN BIOGEOGRÁFICA } & EUROSIBERIANA \\
\hline & & MEDITERRÁNEA \\
\hline \multirow{2}{*}{2} & \multirow{2}{*}{ PROVINCIA BIOGEOGRÁFICA } & MEDITERRÁNEO IBÉRICO OCCIDENTAL \\
\hline & & MEDITERRÁNEO IBÉRICO CENTRAL \\
\hline \multirow{3}{*}{3} & \multirow{3}{*}{ SUBPROVINCIA BIOGEOGRÁFICA } & CARPETANO-LEONESA \\
\hline & & LUSO-EXTREMADURENSE \\
\hline & & CASTELLANA \\
\hline \multirow{3}{*}{3} & \multirow{3}{*}{ SECTOR BIOGEOGRÁFICO } & CELTIBÉRICO-ALCARREÑO \\
\hline & & TOLETANO-TAGANO \\
\hline & & MANCHEGO \\
\hline 77 & FAC & OLÓGICOS \\
\hline \multirow{11}{*}{10} & \multirow{11}{*}{ APORTACIÓN MEDIA ANUAL EN hm ${ }^{3}$} & $<50$ \\
\hline & & $50-100$ \\
\hline & & $100-250$ \\
\hline & & $250-500$ \\
\hline & & $500-1.000$ \\
\hline & & $1.000-2.000$ \\
\hline & & $2.000-3.000$ \\
\hline & & $3.000-5.000$ \\
\hline & & $5.000-7.000$ \\
\hline & & $7.000-10.000$ \\
\hline & & $>10.000$ \\
\hline \multirow{6}{*}{10} & \multirow{6}{*}{$\begin{array}{l}\text { APORTACIÓN MEDIA ANUAL EN hm }{ }^{3} \\
\text { (CLASES) }\end{array}$} & $<250$ \\
\hline & & $250-500$ \\
\hline & & $500-1000$ \\
\hline & & $1.000-3.000$ \\
\hline & & $3.000-7.000$ \\
\hline & & $>7.000$ \\
\hline \multirow{9}{*}{1} & \multirow{9}{*}{ TAMAÑO DE LA CUENCA } & A. $>500 \mathrm{~km}^{2}$ \\
\hline & & B. $500-1.000 \mathrm{~km}^{2}$ \\
\hline & & C. $1.000-2.000 \mathrm{~km}^{2}$ \\
\hline & & D. $2.000-5.000 \mathrm{~km}^{2}$ \\
\hline & & E. $5.000-10.000 \mathrm{~km}^{2}$ \\
\hline & & F. $10.000-25.000 \mathrm{~km}^{2}$ \\
\hline & & G.25.000-50.000 km² \\
\hline & & H. $50.000-75.000 \mathrm{~km}^{2}$ \\
\hline & & I. $>75.000 \mathrm{~km}^{2}$ \\
\hline \multirow{5}{*}{1} & \multirow{5}{*}{$\begin{array}{c}\text { TAMAÑO DE LA CUENCA } \\
\text { (CLASES) }\end{array}$} & $\mathrm{A}+\mathrm{B}$ \\
\hline & & $\mathrm{C}+\mathrm{D}+\mathrm{E}$ \\
\hline & & $\mathrm{F}+\mathrm{G}$ \\
\hline & & H. \\
\hline & & I. \\
\hline \multirow{2}{*}{6} & \multirow{2}{*}{ ESTACIONALIDAD DE LOS CAUDALES } & PERMANENTE \\
\hline & & ESTACIONAL \\
\hline
\end{tabular}




\begin{tabular}{|c|c|c|}
\hline PESO & FACTORES & PARÁMETROS \\
\hline \multirow{5}{*}{2} & \multirow{5}{*}{ CALIDAD DEL AGUA } & MUY MALA \\
\hline & & MALA \\
\hline & & REGULAR \\
\hline & & BUENA \\
\hline & & MUY BUENA \\
\hline \multirow{3}{*}{2} & \multirow{3}{*}{ CALIDAD DEL AGUA (CLASES) } & MALA-MUY MALA \\
\hline & & REGULAR \\
\hline & & BUENA-MUY BUENA \\
\hline \multirow{4}{*}{1} & \multirow{4}{*}{ GRADO DE REGULACIÓN } & $<25 \%$ \\
\hline & & $25-50 \%$ \\
\hline & & $50-75 \%$ \\
\hline & & $>75 \%$ \\
\hline \multirow{2}{*}{1} & \multirow{2}{*}{ (CLASES) } & $<50 \%$ \\
\hline & & $>50 \%$ \\
\hline \multirow{4}{*}{2} & \multirow{4}{*}{$\begin{array}{l}\text { APORTACIÓN REAL/APORTACIÓN } \\
\text { NATURAL }\end{array}$} & $<25 \%$ \\
\hline & & $25-50 \%$ \\
\hline & & $50-75 \%$ \\
\hline & & $>75 \%$ \\
\hline \multirow{2}{*}{2} & \multirow{2}{*}{ (CLASES) } & $<50 \%$ \\
\hline & & $>50 \%$ \\
\hline \multirow{4}{*}{2} & \multirow{4}{*}{$\begin{array}{l}\text { ALTERACIÓN EN LA DISTRIBUCIÓN } \\
\text { ESTACIONAL DE APORTACIONES }\end{array}$} & NULA-MUY BAJA \\
\hline & & BAJA \\
\hline & & MEDIA \\
\hline & & ALTA \\
\hline \multirow{2}{*}{2} & \multirow{2}{*}{$\begin{array}{l}\text { ALTERACIÓN EN LA DISTRIBUCIÓN } \\
\text { ESTACIONAL DE APORTACIONES }\end{array}$} & NULA-BAJA \\
\hline & & MEDIA-ALTA \\
\hline \multirow{5}{*}{10} & \multirow{5}{*}{ GRADO DE INUNDACIÓN } & TRAMO NO EMBALSADO \\
\hline & & AZUDES \\
\hline & & PEQUEÑOS EMBALSES (1-50 hm3) \\
\hline & & MEDIANOS EMBALSES (50-250 hm3) \\
\hline & & GRANDES EMBALSES (>250 hm3) \\
\hline \multirow{4}{*}{10} & \multirow{4}{*}{ EMBALSES-AZUDES (SUPERFICIE) } & AZUDES \\
\hline & & PEQUEÑOS EMBALSES (<250 ha) \\
\hline & & MEDIANOS EMBALSES (250-1.000 ha) \\
\hline & & GRANDES EMBALSES (>1.000 hA) \\
\hline \multirow{2}{*}{15} & \multirow{2}{*}{ GRADO DE INUNDACIÓN (CLASES) } & TRAMO EMBALSADO \\
\hline & & TRAMO NO EMBALSADO \\
\hline 50 & \multicolumn{2}{|c|}{ FACTORES GEOMORFOLÓGICOS Y TOPOGRÁFICOS } \\
\hline \multirow{9}{*}{1} & & $<0,5 \mathrm{~m} / \mathrm{km}$ \\
\hline & & $0,5-1 \mathrm{~m} / \mathrm{km}$ \\
\hline & & $1-2,5 \mathrm{~m} / \mathrm{km}$ \\
\hline & & $2,5-5 \mathrm{~m} / \mathrm{km}$ \\
\hline & PENDIENTE MEDIA DEL CAUCE & $5-10 \mathrm{~m} / \mathrm{km}$ \\
\hline & & $10-20 \mathrm{~m} / \mathrm{km}$ \\
\hline & & $20-50 \mathrm{~m} / \mathrm{km}$ \\
\hline & & $50-100 \mathrm{~m} / \mathrm{km}$ \\
\hline & & $>100 \mathrm{~m} / \mathrm{km}$ \\
\hline
\end{tabular}




\begin{tabular}{|c|c|c|}
\hline PESO & FACTORES & PARÁMETROS \\
\hline \multirow{5}{*}{1} & \multirow{5}{*}{ PENDIENTE MEDIA DEL CAUCE (CLASES) } & $<1 \mathrm{~m} / \mathrm{km}$ \\
\hline & & $1-5 \mathrm{~m} / \mathrm{km}$ \\
\hline & & $5-20 \mathrm{~m} / \mathrm{km}$ \\
\hline & & $20-50 \mathrm{~m} / \mathrm{km}$ \\
\hline & & $>50 \mathrm{~m} / \mathrm{km}$ \\
\hline \multirow{8}{*}{1} & \multirow{8}{*}{ PENDIENTE MEDIA DEL VALLE } & $<1 \mathrm{~m} / \mathrm{km}$ \\
\hline & & $1-2,5 \mathrm{~m} / \mathrm{km}$ \\
\hline & & $2,5-5 \mathrm{~m} / \mathrm{km}$ \\
\hline & & $5-10 \mathrm{~m} / \mathrm{km}$ \\
\hline & & $10-20 \mathrm{~m} / \mathrm{km}$ \\
\hline & & $20-50 \mathrm{~m} / \mathrm{km}$ \\
\hline & & $50-100 \mathrm{~m} / \mathrm{km}$ \\
\hline & & $>100 \mathrm{~m} / \mathrm{km}$ \\
\hline \multirow{5}{*}{1} & \multirow{5}{*}{ PENDIENTE MEDIA DEL VALLE (CLASES) } & $<1 \mathrm{~m} / \mathrm{km}$ \\
\hline & & $1-5 \mathrm{~m} / \mathrm{km}$ \\
\hline & & $5-20 \mathrm{~m} / \mathrm{km}$ \\
\hline & & $20-50 \mathrm{~m} / \mathrm{km}$ \\
\hline & & $>50 \mathrm{~m} / \mathrm{km}$ \\
\hline \multirow{3}{*}{4} & \multirow{3}{*}{ TIPO DE CANAL } & MEANDRIFORME \\
\hline & & RECTILÍNEO \\
\hline & & TRENZADO \\
\hline \multirow{2}{*}{2} & \multirow{2}{*}{ MOVILIDAD DEL CANAL } & DIVAGANTE \\
\hline & & NO DIVAGANTE \\
\hline \multirow{6}{*}{2} & \multirow{6}{*}{ NIVEL ALTITUDINAL } & $<250 \mathrm{~m}$ \\
\hline & & $250-500 \mathrm{~m}$ \\
\hline & & $500-750 \mathrm{~m}$ \\
\hline & & $750-1.000 \mathrm{~m}$ \\
\hline & & $1.000-1.500 \mathrm{~m}$ \\
\hline & & $1.500-2000 \mathrm{~m}$ \\
\hline \multirow{3}{*}{2} & \multirow{3}{*}{ NIVEL ALTITUDINAL (CLASES) } & $<500 \mathrm{~m}$ \\
\hline & & $500-1.000 \mathrm{~m}$ \\
\hline & & $1.000-1.500 \mathrm{~m}$ \\
\hline \multirow{11}{*}{8} & \multirow{11}{*}{ MORFOLOGÍA DEL FONDO DE VALLE } & A. HOCES, CAÑONES, GARGANTAS Y CONGOSTOS \\
\hline & & $\begin{array}{l}\text { B. FONDOS DE VALLE ENCAJADOS EN ZONAS MONTANAS Y } \\
\text { SIERRAS }\end{array}$ \\
\hline & & C.FONDOS DE VALLE ENCAJADOS EN PARAMERAS \\
\hline & & D. FONDOS DE VALLE ENCAJADOS EN PÁRAMOS \\
\hline & & E. FONDOS DE VALLE ENCAJADOS EN LLANURAS Y CAMPIÑAS \\
\hline & & $\begin{array}{l}\text { F. FONDOS DE VALLE ENCAJADOS EN PIEDEMONTES Y } \\
\text { PENILLANURAS }\end{array}$ \\
\hline & & G.OTROS FONDOS DE VALLE ENCAJADOS \\
\hline & & $\begin{array}{l}\text { H. FONDOS DE VALLE ABIERTOS EN ZONAS MONTANAS Y } \\
\text { SIERRAS }\end{array}$ \\
\hline & & I. FONDOS DE VALLE ABIERTOS EN LLANURAS Y CAMPIÑAS \\
\hline & & J. FONDOS DE VALLE ABIERTOS EN PÁRAMOS \\
\hline & & K. GRANDES LLANURAS ALUVIALES \\
\hline \multirow{2}{*}{8} & \multirow{2}{*}{ (CLASES) } & A-B-C-D-E+F+G \\
\hline & & G-H-I-J+K \\
\hline
\end{tabular}


continuação

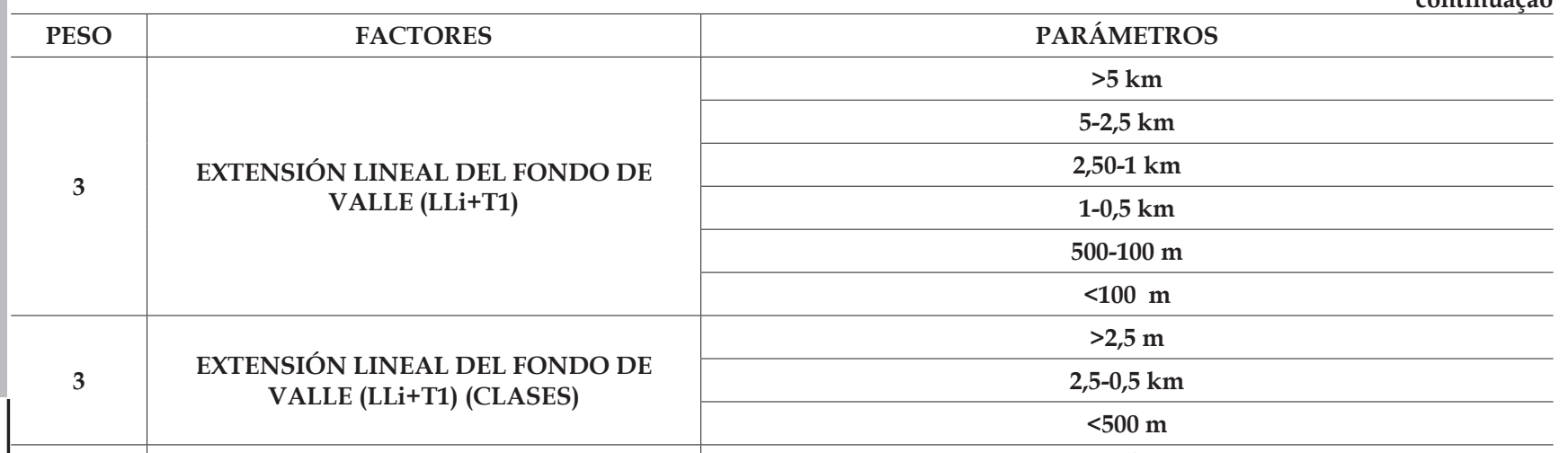

A. SIERRAS GRANÍTICO-GNEíSICAS

B. SIERRAS Y SERRANÍAS CUARCÍTICAS

C. SIERRAS ESQUISTOSAS O PIZARROSAS

D. SIERRAS CALCÁREO-DOLOMÍTICAS

\begin{tabular}{l|c}
\multirow{4}{*}{ MARCO GEOMORFOLÓGICO } & E. PARAMERAS \\
\cline { 3 - 3 } & F. PIEDEMONTES \\
\cline { 3 - 3 } & G. PENILLANURAS
\end{tabular}

\begin{tabular}{|c|c|c|}
\hline & & \multirow[b]{2}{*}{ H. CUENCAS TERCIARIAS DETRÍTICAS O ARCILLOSAS } \\
\hline & & \\
\hline & & I. CUENCAS TERCIARIAS YESÍFERAS-CARBONATADAS \\
\hline & & J. PÁRAMOS TERCIARIOS \\
\hline \multirow{3}{*}{4} & \multirow{3}{*}{ (CLASES) } & A-B-C-D \\
\hline & & E-F-G \\
\hline & & H-I-J \\
\hline \multirow{3}{*}{4} & \multirow{3}{*}{ LITOLOGÍA DE LA CAJA } & MATERIALES DE CUENCA SEDIMENTARIA \\
\hline & & MATERIALES DE PLATAFORMA \\
\hline & & MATERIALES DE ZÓCALO \\
\hline \multirow{3}{*}{2} & \multirow{3}{*}{$\begin{array}{l}\text { MATERIALES DOMINANTES EN LA } \\
\text { LLANURA DE INUNDACIÓN }\end{array}$} & ARCILLAS-ARENAS \\
\hline & & GRAVAS-CANTOS \\
\hline & & SUSTRATOS HETEROGÉNEOS \\
\hline
\end{tabular}

\begin{tabular}{|c|c|c|}
\hline \multirow{9}{*}{3} & \multirow{9}{*}{ TIPO DE VEGETACIÓN $1^{\text {a }}$ BANDA } & ABEDULARES \\
\hline & & ALAMEDAS-CHOPERAS \\
\hline & & SAUCEDAS ARBÓREAS \\
\hline & & SAUCEDAS ARBORESCENTES \\
\hline & & FRESNEDAS DE Fraxinus angustifolia \\
\hline & & FRESNEDAS DE Fraxinus excelsior \\
\hline & & TARAYALES \\
\hline & & TAMUJARES \\
\hline & & VEGETACIÓN PALUSTRE \\
\hline \multirow{9}{*}{3} & \multirow{9}{*}{ TIPO DE VEGETACIÓN $2^{a}$ BANDA } & ABEDULARES \\
\hline & & ALAMEDAS-CHOPERAS \\
\hline & & SAUCEDAS ARBÓREAS \\
\hline & & SAUCEDAS ARBORESCENTES \\
\hline & & FRESNEDAS \\
\hline & & TARAYALES \\
\hline & & TAMUJARES \\
\hline & & PLANTACIONES DE Eucalyptus \\
\hline & & COMUNIDADES HERBÁCEAS \\
\hline
\end{tabular}




\begin{tabular}{|c|c|c|}
\hline PESO & FACTORES & PARÁMETROS \\
\hline \multirow{18}{*}{3} & \multirow{18}{*}{ COMUNIDADES VEGETALES $1^{a}$ BANDA } & ABEDULARES DE Betula alba \\
\hline & & ALAMEDAS DE Populus trermula \\
\hline & & ALAMEDAS DE Populus alba \\
\hline & & SAUCEDAS DE Salix alba \\
\hline & & CHOPERAS DE Populus nigra \\
\hline & & ALISEDAS \\
\hline & & FRESNEDAS DE Fraxinus angustifolia \\
\hline & & FRESNEDAS DE Fraxinus excelsior \\
\hline & & TARAYALES DE Tamarix gallica Y Tamarix africana \\
\hline & & SAUCEDAS ARBORESCENTES DE Salix atrocinerea \\
\hline & & SAUCEDAS ARBORESCENTES DE Salix salvifolia \\
\hline & & SAUCEDAS ARBORESCENTES DE Salix salvifolia Y S. atrocinerea \\
\hline & & SAUCEDAS BASÓFILAS \\
\hline & & SAUCEDAS ARBORESCENTES MIXTAS \\
\hline & & CARRIZALES-ESPADAÑALES \\
\hline & & HERBZALES DE Carex spp. \\
\hline & & COMUNIDADES DE Sparganium \\
\hline & & JUNCALES DE Scirpus lacustris \\
\hline \multirow{14}{*}{3} & \multirow{14}{*}{ COMUNIDADES VEGETALES $2^{\mathrm{a}}$ BANDA } & ABEDULARES DE Betula alba \\
\hline & & ALAMEDAS DE Populus alba \\
\hline & & SAUCEDAS DE Salix alba \\
\hline & & CHOPERAS DE Populus nigra \\
\hline & & ALISEDAS \\
\hline & & FRESNEDAS DE Fraxinus angustifolia \\
\hline & & TARAYALES DE Tamarix gallica Y Tamarix africana \\
\hline & & SAUCEDAS ARBORESCENTES DE Salix atrocinerea \\
\hline & & SAUCEDAS ARBORESCENTES DE Salix salvifolia \\
\hline & & SAUCEDAS ARBORESCENTES DE Salix salvifolia Y S. atrocinerea \\
\hline & & SAUCEDAS ARBORESCENTES MIXTAS \\
\hline & & PLANTACIONES DE Eucalyptus \\
\hline & & COMUNIDADES DE VEGETACIÓN PALUSTRE \\
\hline & & COMUNIDADES HERBÁCEAS \\
\hline \multirow{4}{*}{3} & \multirow{4}{*}{ ESTRUCTURA DE LA VEGETACIÓN } & ARBÓREA \\
\hline & & ARBORESCENTE \\
\hline & & ARBUSTIVA \\
\hline & & SUBARBUSTIVA-HERBÁCEA \\
\hline \multirow{2}{*}{3} & \multirow{2}{*}{$\begin{array}{l}\text { ESTRUCTURA DE LA VEGETACIÓN } \\
\text { (CLASES) }\end{array}$} & HERBÁCEA \\
\hline & & LEÑOSA \\
\hline \multirow{5}{*}{2} & \multirow{5}{*}{ EXTENSIÓN DE LA VEGETACIÓN } & $<10 \mathrm{~m}$ \\
\hline & & $10-25 \mathrm{~m}$ \\
\hline & & $25-50 \mathrm{~m}$ \\
\hline & & $50-100 \mathrm{~m}$ \\
\hline & & $>100 \mathrm{~m}$ \\
\hline
\end{tabular}


Pedro Molina Holgado et al.

continuação

\begin{tabular}{|c|c|c|}
\hline PESO & FACTORES & PARÁMETROS \\
\hline \multirow{2}{*}{2} & \multirow{2}{*}{ EXTENSIÓN DE LA VEGETACIÓN } & $<50 \mathrm{~m}$ \\
\hline & & $>50 \mathrm{~m}$ \\
\hline \multirow{4}{*}{2} & \multirow{4}{*}{$\begin{array}{l}\text { CONTINUIDAD LINEAL DE LA } \\
\text { VEGETACIÓN EN \% }\end{array}$} & $0-25 \%$ \\
\hline & & $25-50 \%$ \\
\hline & & $50-75 \%$ \\
\hline & & $>75 \%$ \\
\hline \multirow{2}{*}{2} & \multirow{2}{*}{$\begin{array}{l}\text { CONTINUIDAD LINEAL DE LA } \\
\text { VEGETACIÓN EN \% (CLASES) }\end{array}$} & $<50 \%$ \\
\hline & & $>50 \%$ \\
\hline \multirow{4}{*}{2} & \multirow{4}{*}{ COMPLEJIDAD ESTRUCTURAL } & NIVEL 1: doseles simples con tramos sin vegetación \\
\hline & & NIVEL 2: doseles simples \\
\hline & & NIVEL 3 doseles simples con sotos complejos \\
\hline & & NIVEL 4: sotos complejos-pluriestratos \\
\hline \multirow{2}{*}{2} & \multirow{2}{*}{ (CLASES) } & NIVEL $1+2$ \\
\hline & & NIVEL $3+4$ \\
\hline \multirow{5}{*}{2} & \multirow{5}{*}{ CONEXIÓN RIBERA-VEGA-VERTIENTE } & MUY ALTA (>75\%) \\
\hline & & ALTA $(50-75 \%)$ \\
\hline & & MEDIA (25-50\%) \\
\hline & & BAJA (5-25\%) \\
\hline & & MUY BAJA (<5\%) \\
\hline \multirow{2}{*}{2} & \multirow{2}{*}{ (CLASES) } & $>50 \%$ \\
\hline & & $<50 \%$ \\
\hline 40 & & ISTEMA \\
\hline \multirow{7}{*}{10} & \multirow{7}{*}{ TIPO DE USO } & PASTIZALES \\
\hline & & FORESTAL \\
\hline & & REGADÍO \\
\hline & & SECANO \\
\hline & & MEZCLA DE REGADÍOS Y SECANOS \\
\hline & & EXTRACCIONES DE ÁRIDOS \\
\hline & & RECREATIVOS \\
\hline \multirow{3}{*}{10} & \multirow{3}{*}{ TIPO DE REGADÍO } & TRADICIONAL-HISTÓRICO \\
\hline & & MODERNOS-INNOVACIÓN \\
\hline & & MEZCLAS \\
\hline \multirow{12}{*}{5} & \multirow{12}{*}{ TIPO DE CULTIVO } & A.PASTOS NATURALES, ARTIFICIALES O MEJORADOS \\
\hline & & B. CEREALES DE INVIERNO \\
\hline & & C. MAÍZ GRANO-FORRAJERO \\
\hline & & D. ALFALFA \\
\hline & & E. OTROS CULTIVOS HERBÁCEOS FORRAJEROS \\
\hline & & F. GIRASOL \\
\hline & & G. HORTALIZAS \\
\hline & & H. TABACO Y OTROS CULTIVOS INDUSTRIALES \\
\hline & & I. FRUTALES \\
\hline & & J. OLIVAR, VIÑEDO Y OTROS \\
\hline & & K. CHOPERAS \\
\hline & & L. FORESTAL (SIN USO) \\
\hline \multirow{3}{*}{5} & \multirow{3}{*}{ (CLASES) } & HERBÁCEOS (A.B.C.D,E, F, G, H) \\
\hline & & LEÑOSOS (I, J, K,) \\
\hline & & SIN USO (L) \\
\hline
\end{tabular}




\begin{tabular}{|c|c|c|}
\hline PESO & FACTORES & PARÁMETROS \\
\hline \multirow{4}{*}{4} & \multirow{4}{*}{ GRADO DE INTENSIDAD PRODUCTIVA } & ALTO \\
\hline & & MEDIO \\
\hline & & BAJO \\
\hline & & MUY BAJO \\
\hline \multirow{4}{*}{6} & \multirow{4}{*}{$\begin{array}{l}\text { TAMAÑO DE LA EXPLOTACIÓN Y } \\
\text { PARCELARIO }\end{array}$} & GRANDE \\
\hline & & MEDIANO \\
\hline & & PEQUEÑO \\
\hline & & NO APRECIABLE \\
\hline 25 & \multicolumn{2}{|c|}{ TRAMAS E INFRAESTRUCTURAS RURARLES } \\
\hline \multirow{4}{*}{10} & \multirow{4}{*}{ CANALES } & A. BAJO DESARROLLO \\
\hline & & B. DESARROLLO MEDIO \\
\hline & & C. AMPLIO DESARROLLO \\
\hline & & NO APRECIABLE \\
\hline \multirow{4}{*}{10} & \multirow{4}{*}{ VIARIO } & A. BAJO DESARROLLO \\
\hline & & B. DESARROLLO MEDIO \\
\hline & & C. AMPLIO DESARROLLO \\
\hline & & NO APRECIABLE \\
\hline \multirow{4}{*}{5} & \multirow{4}{*}{ TRANSPORTE DE ENERGÍA } & A. BAJO DESARROLLO \\
\hline & & B. DESARROLLO MEDIO \\
\hline & & C. AMPLIO DESARROLLO \\
\hline & & NO APRECIABLE \\
\hline 60 & & \\
\hline \multirow{3}{*}{25} & \multirow{3}{*}{ TRAMOS URBANOS } & TRAMO URBANO \\
\hline & & TRAMO SUBURBANO \\
\hline & & TRAMO NO URBANO \\
\hline \multirow{4}{*}{6} & \multirow{4}{*}{ NÚCLEOS TRADICIONALES } & AUSENCIA \\
\hline & & ESCASOS \\
\hline & & NUMEROSOS \\
\hline & & NO APRECIABLE \\
\hline \multirow{4}{*}{3} & \multirow{4}{*}{ NÚCLEOS TRADICIONALES TIPO } & CIUDAD \\
\hline & & PUEBLO-ALDEA \\
\hline & & HÁBITAT DISPERSO \\
\hline & & NO APRECIABLE \\
\hline \multirow{4}{*}{4} & \multirow{4}{*}{$\begin{array}{l}\text { NÚCLEOS TRADICIONALES } \\
\text { LOCALIZACIÓN }\end{array}$} & VEGA \\
\hline & & VERTIENTE \\
\hline & & CORNISA \\
\hline & & NO APRECIABLE \\
\hline \multirow{3}{*}{6} & \multirow{3}{*}{ NÚCLEOS DE COLONIZACIÓN } & ESCASOS \\
\hline & & NUMEROSOS \\
\hline & & NO APRECIABLE \\
\hline \multirow{3}{*}{4} & \multirow{3}{*}{ NÚCLEOS DE COLONIZACIÓN TIPO } & NIVEL 1 \\
\hline & & NIVEL 2 \\
\hline & & NO APRECIABLE \\
\hline \multirow{4}{*}{4} & & AUSENCIA \\
\hline & PORI AMLENTO DISEMINADO & ESCASOS \\
\hline & & NUMEROSOS \\
\hline & & NO APRECIABLE \\
\hline
\end{tabular}




\begin{tabular}{c|c|c}
\hline PESO & FACTORES & PARÁMETROS \\
\hline \multirow{3}{*}{4} & \multirow{2}{*}{ POBLAMIENTO DISEMINADO TIPO } & NIVEL 1: PEQUEÑAS CASAS DE LABRANZA \\
\cline { 3 - 3 } & & NIVEL 2:GRANDES CASAS DE LABRANZA \\
\cline { 3 - 3 } & \multirow{3}{*}{4} & GRANDES SUPERFICIES PRODUCTIVAS, \\
\cline { 3 - 3 } & EQUIPAMIENTOS Y OTRAS DOTACIONES & A. BAJO DESARROLLO \\
\cline { 3 - 3 } & & B. DESARROLLO MEDIO \\
\cline { 3 - 3 } & & C. AMPLIO DESARROLLO \\
\hline
\end{tabular}

\section{RESULTADOS}

\subsection{Los tipos de paisaje de los ríos Tajo y Jarama}

La representación gráfica del análisis de similitud (Figs. 5y 6) ha permitidoidentificar 12 grupos o tipos de paisajes fluviales en función de los criterios considerados en la caracterización, existiendo diferencias estadísticas significativas $(K=166,74$; $p<0,05 ; n=38$ ) en el conjunto de los mismos. Este análisis ha permitido reconocer, en una primera aproximación, 3 macrogrupos: vegas (Fertil lowlands), riberas/gargantas (Fluvial shores and Gorges) y embalses (Dams and Reservoirs). El primero reúne todas las vegas del Tajo entre el embalse de Valdecañas (Cáceres) y el embalse de Entrepeñas (Guadalajara), el segundo los valles más angostos que carecen de vega y en el que en ocasiones se forman gargantas y cañones $\mathrm{y}$, finalmente, el tercero, que agrupa a todos los embalses mayores de 3 $\mathrm{hm}^{3}$ de capacidad.

Estos macrogrupos integran varios grupos. El número de tramos por grupo varía considerablemente $\mathrm{y}$, por tanto, la extensión y representación paisajística de cada uno de los grupos es desigual.

El Grupo 2 (Grandes vegas del Tajo medio), el más extenso, reúne 12 tramos que agrupan las grandes vegas de tramo medio del valle del Tajo aguas abajo de la ciudad de Toledo. Dentro de este tipo de paisaje se reconocen dos subtipos, aunque el conjunto de los tramos no muestran diferencias estadísticamente significativas $(K=0,745 ; p>0,05 ; n=12)$.

Los Grupos 4, 6, 9, 10y 11, por el contrario, están formados por un solo tramo. Esta característica denota condiciones de singularidad que pueden ser atribuidas en algunos casos al tamaño de la muestra y no a auténticas condiciones de singularidad paisajística si se considera el conjunto de los ríos ibéricos; sin embargo, sí existe esa singularidad en el caso del Grupo 10, en el que integra un paisaje que tendría más amplia distribución en condiciones naturales pero que se ha visto drásticamente reducido por la acción antrópica. En efecto, las gargantas del Tajo en el piedemonte de los Montes de Toledo son paisajes de amplia distribución potencial en la Cuenca Hidrográfica del Tajo, aguas abajo del embalse de Valdecañas, precisamente el límite inferior del área de estudio. En condiciones naturales, estos paisajes ocuparían un extenso sector del río Tajo en Extremadura aunque la construcción de presas en la mayor parte del recorrido del río ha eliminado muchas gargantas y valles encajados similares a los existentes entre el embalse de Valdecañas y El Puente del Arzobispo.

En los restantes casos, la singularidad es genuina y puede atribuirse a factores diversos, aunque el hecho urbano es posiblemente la causa principal de la misma en la zona de estudio. La presencia de importantes núcleos riberiegos como las ciudades de Talavera de la Reina, Toledo o Aranjuez introduce rasgos de singularidad en las márgenes del Tajo que generan 
paisajes particulares con características comunes a los ámbitos en los que se sitúan pero que se diferencian bien de éstos por poseer específicidades resultado de tratamientos y usos que derivan de su condición urbana o suburbana. No en vano, estas tres ciudades son las capitales del Tajo, los núcleos más poblados de estas riberas desde hace siglos.

Debe destacarse especialmente la importancia de estos paisajes singulares, únicos e irrepetibles, y su gestión debería considerar siempre criterios de calidad paisajística.

Cuadro 3 - Macrogrupos y tipos de paisaje identificados a partir de análisis de similitud

\begin{tabular}{|c|c|}
\hline \multicolumn{2}{|c|}{ I. VEGAS (Fertile lowlands) } \\
\hline • & 1. GRANDES VEGAS DEL BAJO JARAMA. Tramos J1, J2 \\
\hline \multirow[t]{2}{*}{$\bullet$} & 2. GRANDES VEGAS DEL TAJO MEDIO. \\
\hline & $\begin{array}{ll} & \text { 2.1 GRANDES VEGAS: Tramos } 6,7,8,12,13,14,16,17,18,19 \\
\text { - } \quad 2.2 \text { VEGAS DE LA PUEBLA DE MONTALBÁN-EL CARPIO: Tramos 9, } 10 .\end{array}$ \\
\hline \multirow[t]{2}{*}{$\bullet$} & 3. VEGAS MEDIAS DE MADRID Y GUADALAJARA: Tramos $21,22,23,24$ \\
\hline & $\begin{array}{l}\text { 3.1. VEGAS DE OREJA-FUENTIDUEÑA } \\
\text { 3.2. VEGAS DE ESTREMERA }\end{array}$ \\
\hline • & 4. VEGA DE AZUTÁN-EL PUENTE DEL ARZOBISPO: Tramo 3 \\
\hline$\bullet$ & 5. VEGA DE ARANJUEZ: Tramo 20 \\
\hline • & 6. PEQUEÑAS VEGAS DE LA BAJA ALCARRIA: Tramos 26, 27 \\
\hline$\bullet$ & 7. VEGA DE TALAVERA DE LA REINA: Tramo 5 \\
\hline \multicolumn{2}{|c|}{ II. RIBERAS Y GARGANTAS (Fluvial shores and Gorges) } \\
\hline • & 9. HOCES, CAÑONES Y GARGANTAS DEL ALTO TAJO: Tramos 34, 35, 36 \\
\hline$\bullet$ & 10. GARGANTA DEL PIEDEMONTE DE VALDECAÑAS: Tramo 2 \\
\hline$\bullet$ & 11. RÍO TAJO EN TOLEDO: Tramo 15 \\
\hline \multicolumn{2}{|c|}{ III. EMBALSES (Dams and Reservoirs) } \\
\hline$\bullet$ & 12. EMBALSES: Tramos 1, 4, 11, 25, 28, 29 \\
\hline
\end{tabular}

Org.: Autores

Figura 5 - Análisis de similitud UPGMA de caracterización de paisajes fluviales en la Cuenca Hidrográfica del Tajo

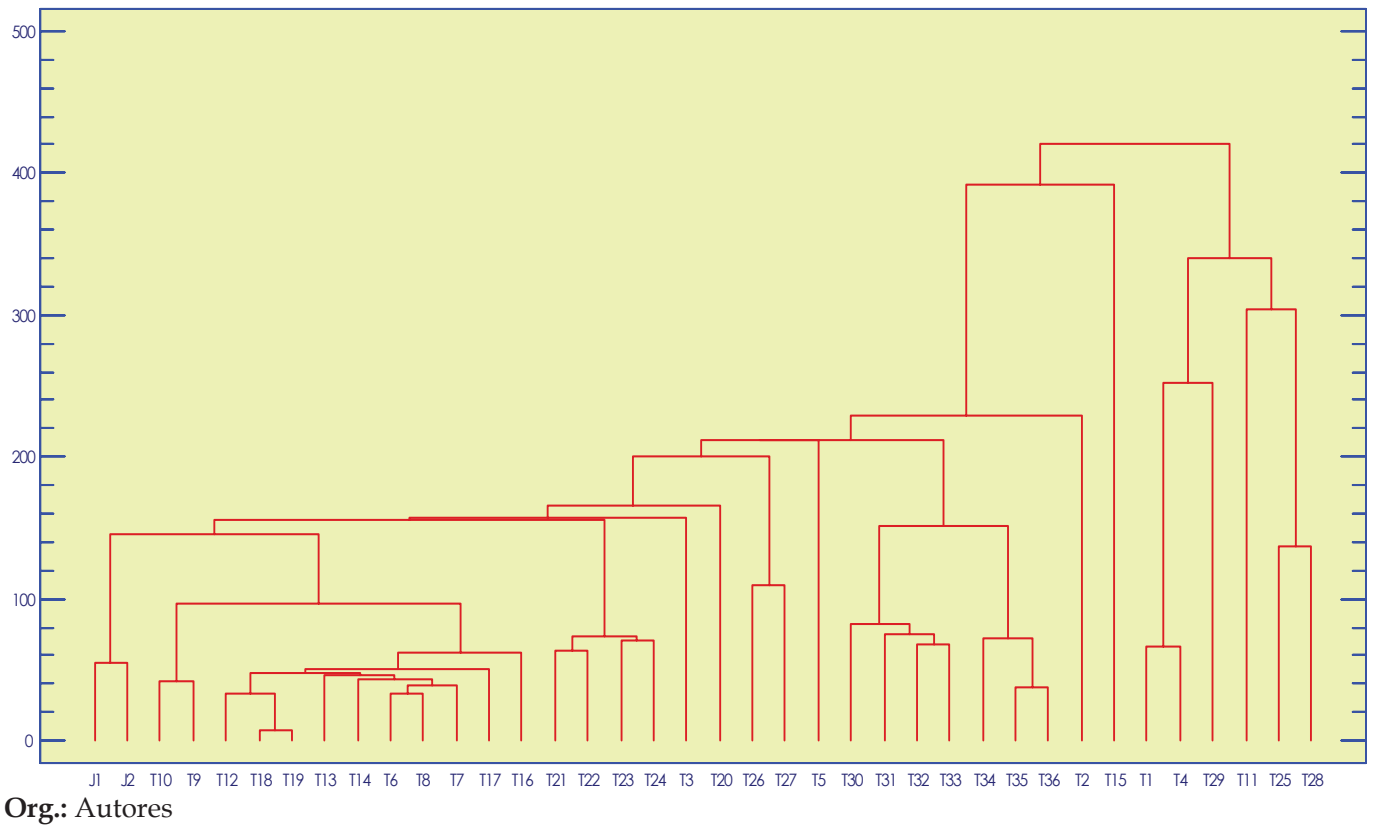


Pedro Molina Holgado et al.

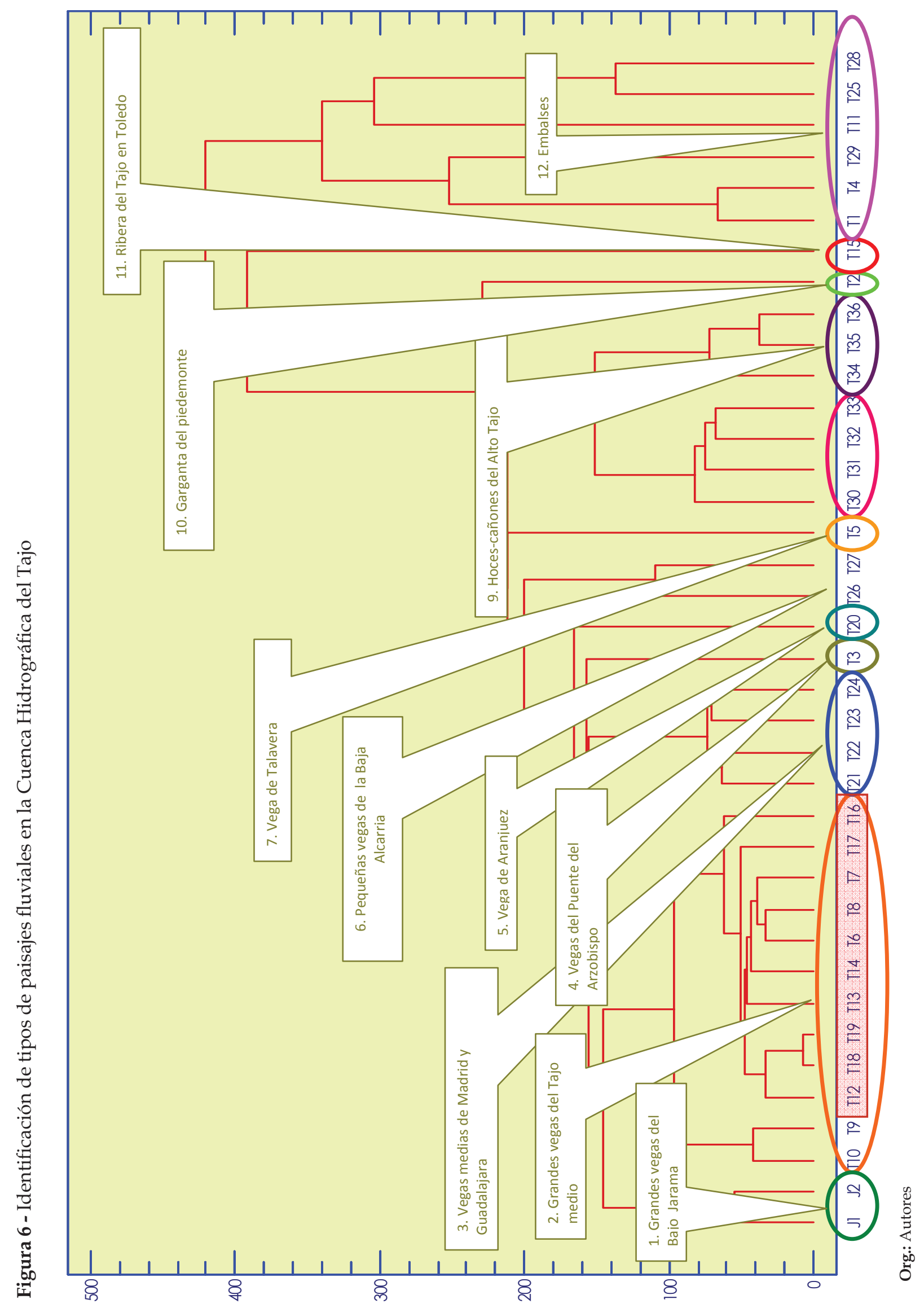




\section{REFERENCIAS}

ANTROP, M. Why landscapes of the past are important for the future. Landscape and Urban Planning, v. 70, n. 1-2, p. 21-34, 2005.

BETHEMONT, J. Les grands fleuves: Entre nature et société. Paris: Armand Colin, 1999.

BETHEMONT, J.; PIEGAY, H. Les paysages des cours d'eau: structure, perception, gestion. Revue de Géographie de Lyon, v. 73, n. 4, p. 271-272, 1998.

BETHEMONT, J.; HONEGGER-RIVIÈRE, A.; LE LAY, Y.-F. Les paysages des eaux douces. Géoconfluences, Le paysage dans tous ses états, 2006. Disponível em: http://geoconfluences. ens...r/doc/transv/paysage/PaysageScient2. htm.

CARAPINHA, A. Los tiempos del paisaje. In: MADERUELO, J. (Ed.): Paisaje e Historia. Madrid: CDAN-Abada Editores, 2009.

CASIMIRO, C.; SÁNCHEZ, J.M.; COSTILLO, E. Patterns of structural complexity and human disturbance of riparian vegetation in agricultural landscapes of a Mediterranean area. Agriculture Ecosystems \& Environment, v. 95, p. 495-507, 2003.

CONFEDERACIÓN HIDROGRÁFICA DEL TAJO. La Cuenca del Tajo en cifras. Confederación Hidrográfica del Tajo. Madrid: Ministerio de Medio Ambiente, 2002.

COUNCIL OF EUROPE. Fifth meeting of the Workshops of the Council of Europe for the implementation of the European Landscape Convention. Landscape quality objectives: from theory to practice. European spatial planning and landscape, 2006.436 p. v. 84.

DÍAZ BEA, E.; OLLERO, A. Metodología para la clasificación geomorfológica de los ríos de la cuenca del Ebro. Geographicalia, v. 47, p. 23-45, 2005.

GONZÁLEZ, M.A.; DE LA LASTRA, I. La urbanización y su efecto en los ríos. Mesa de Trabajo. Estrategia Nacional de Restauración de Ríos y Riberas. Ministerio de Medio Ambiente, 2007. Disponível em: www.mma.es

GONZÁLEZ DEL TÁNAGO, M.; GARCÍA DE JALÓN, D. Propuesta para la caracterización jerárquica de los ríos españoles para su clasificación según la Directiva Marco del Agua. Limnetica, v. 25, p. 693-712, 2006.
GONZÁlEZ DEL TÁNAGO, M.; GARCÍA DE JALÓN, D. Restauración de ríos. Guía metodológica para la elaboración de proyectos. Madrid: Ministerio de Medio Ambiente, 2007.

GONZÁLEZ DEL TÁNAGO, M.; GARCÍA DE JALÓN, D.; LARA, F.; GARILLETI, R. Índice RQI para la valoración de riberas fluviales en el contexto de la Directiva Marco del Agua. Ingeniería Civil, v. 143, p.97-108, 2006.

GRANERO MARTÍN, F. Agua y territorio. Arquitectura y paisaje. Sevilla: Universidad de Sevilla, 2003.

HODEN, P.; PURCELL, N. The corrupting Sea. Oxford: Blackwell, 2001.

JIMÉNEZ HERRERO, L.M. Patrimonio natural, cultural y paisajístico. Claves para la sostenibilidad territorial. Observatorio de la Sostenibilidad en España. Madrid: Ministerio de Medio Ambiente, Medio Rural y Marino; Fundación Biodiversidad; Fundación de la Universidad de Alcalá de Henares, 2009.

KNOPF, F.L.; JOHNSON, R.R.; RICH, T.;SMASON, F.B.; SZARO, R.C. Conservation of riparian ecosystems in US. Willson Bulletin, v. 100, p. 272284, 1988.

LAUREANO, P. Atlas de agua. Los conocimientos tradicionales para combatir la desertificación. Barcelona: UNESCO-Laia Libros, 2005.

MALANSON, G.P. Riparian Landscapes. Cambridge: Cambridge University Press, 1993.

MARTÍNEZ DE PISÓN, E. Miradas sobre el paisaje. Madrid: Biblioteca Nueva, 2009.

MARTÍNEZ DE PISÓN, E. El paisaje, entre la ciencia y la cultura. Laberintos, v. 21, 2010.

MATA OLMO, R. Un concepto de paisaje para la gestión sostenible del territorio. In: MATA R.; TARROJA, A. (Orgs.): El paisaje y la gestión del territorio. Criterios paisajísticos en la ordenación del territorio y el urbanismo. Barcelona: Diputación de Barcelona, 2006.

MATA OLMO, R.; SANZ HERRÁIZ, C. (Eds.). Atlas de los Paisajes de España. Madrid: Centro de publicaciones del Ministerio de Medio Ambiente, 2003.

MOLINA HOLGADO, P. Los paisajes fluviales de la ribera del Duero en la provincia de Soria. Soria: Fundación Duques de Soria, 2007. Informe inédito. 
MOLINA HOLGADO, P.; BERROCAL MENÁRGUEZ, A.B. Tipología de los paisajes fluviales de los cursos de la Sierra de Guadarrama en la Comunidad de Madrid. In: III CONGRESO DE INGENIERÍA CIVIL, TERRITORIO Y MEDIO AMBIENTE, 2006, Zaragoza. Madrid: $2006 a$.

MOLINA HOLGADO, P.; BERROCAL MENÁRGUEZ, A.B. Los efectos ambientales de la regulación de los cursos de la cabecera de la cuenca del Tajo: la reducción de los bosques aluviales del Tajo-Jarama. In: III CONGRESO DE INGENIERÍA CIVIL, TERRITORIO Y MEDIO AMBIENTE, 2006, Zaragoza. Madrid: 2006b.

MOLINA HOLGADO, P.; SANZ HERRÁIZ, C.; MATA OLMO, R. Los paisajes del Tajo. Madrid: Ministerio de Medio Ambiente, Medio Rural y Marino. Gobierno de España, 2010.

MUNNÉ, A.; SOLÀ, C.; PRAT, N. QBR: un índice rápido para la evaluación de la calidad de los ecosistemas de ribera. Tecnología del Agua, v. 175, p. 20-37, 1998.

NAIMAN, R.J.; DÉCAMPS, H.; POLLOCK, M. The role of riparian corridors in maintaining biodiversity. Ecology Appl., v. 3, p. 209-212, 1993.

OLLERO, A.; ECHEVERRÍA, M.T.; SÁNCHEZ, M.; AURÍA, V.; VALLÁRÍN, D.; MORA, D. Metodología para la tipificación hidromorfológica de los cursos fluviales de Aragón en aplicación de la Directiva Marco del Agua (2006/60/CE). Geographicalia, v. 44, p. 7-25, 2004.

ORTEGA CANTERO, N. Paisaje, Historia y Nación (A propósito del Tableau de la Géographie de la France, de Paul Vidal de La Blache). In: ORTEGA, N. (Ed.): Paisaje, memoria histórica e identidad Nacional. Madrid: UAM Ediciones-Fundación Duques de Soria, 2005.

ORTEGA, N. Visiones históricas del paisaje: entre la ciencia y el sentimiento. In: MARTÍNEZ DE PISÓN,E.; ORTEGA, N. (Eds.): La recuperación del paisaje. Madrid: UAM Ediciones-Fundación Duques de Soria, 2008.

PELLICER, F. Paisajes fluviales de las ciudades de la Red C-6. In: DE LA CALL, P.; PELLICER, F. (Orgs.): Ríos y Ciudades. Zaragoza: Institución Fernando El Católico, 2002.

RENES, J. Paisajes europeos: continuidad y transformaciones. In MADERUELO, J. (Ed.): Paisaje e Historia. Madrid: CDAN-Abada Editores, 2009.
SAULÈ-SORBE, H. "Ante la prueba del motivo artístico: algunas reflexiones sobre la observación en el arte del paisaje". In ORTEGA, N. (Ed.): Imágenes del paisaje. Madrid: UAM EdicionesFundación Duques de Soria, 2006.

SUÁREZ, M.L.; VIDAL-ABARCA, M.R. Aplicación del índice de calidad del bosque de ribera QBR (Munné et al., 1998) a los cauces fluviales de la Cuenca del río Segura. Tecnología del Agua, v. 201, p. 33-45, 2000.

SUÁREZ, M.L.; VIDAL-ABARCA, M.R.; SÁNCHEZ-MONTOYA, M.; ALBA, J.; ÁLVAREZ, M.; AVILÉS, J.; BONADA, N.; CASAS, J.; JÁIMEZCUELLAR, P.; MUNNÉ, A.; PARDO, I.; PRAT, N.; RIBADENEIRA, M.; SALINAS, J.; TORO, M.; VIVAS, S. Las riberas de los ríos mediterráneos y su calidad: el uso del índice QBR. Limnetica, v. 21, n. 3-4, p. 135-148, 2002.

VADILLO, A.; MOLINA HOLGADO P. Los paisajes del río Pisuerga en la ciudad de valladolid: evolución, sostenibilidad y participación ciudadana. In: Paysages de la vie quotidienne. Regards croisés entre la recherche et l'action, 2010. Perpignan (en prensa).

YON, D.; TENDRON, G. Alluvial Forest of Europe. Strasbourg: Council of Europe, 1981. (Nature and Environment Series, 22).

Recebido em: 28/11/10 Aceito em: 28/12/10 